\title{
Active Learning Through The Use Of Virtual Environments
}

\author{
James Mayrose PhD, State University of New York College at Buffalo, USA
}

\begin{abstract}
Immersive Virtual Reality (VR) has seen explosive growth over the last decade. Immersive VR attempts to give users the sensation of being fully immersed in a synthetic environment by providing them with $3 D$ hardware, and allowing them to interact with objects in virtual worlds. The technology is extremely effective for learning and exploration, and has been widely adopted by the military, industry and academia. This current study set out to study the effectiveness of $3 D$ interactive environments on learning, engagement, and preference. A total of 180 students took part in the study where significant results were found regarding preference for this new technology over standard educational practices. Students were more motivated when using the immersive environment than with traditional methods which may translate into greater learning and retention. Larger studies will need to be performed in order to quantify the benefits of this new, cutting edge technology, as it relates to understanding and retention of educational content.
\end{abstract}

Keywords: Virtual Reality; Simulation; Education

\section{INTRODUCTION}

enerally accepted educational theory supports the notion that active learning produces superior mastery of complex ideas versus passive learning. Active learning is defined as hands-on, fully engaged learning versus learning based on passive methods, such as reading text or watching an instructor do something. Virtual reality, the technology that allows computers to present lifelike experiences to users, is an ideal environment for presenting highly interactive active learning experiences that would otherwise be too expensive, unsafe, or otherwise not feasible. Immersive virtual reality, technology wherein a user is presented with a 3-dimensional, real world scaled environment by utilizing a room sized rear-projected screen, is ideal for this type of experience.

The NASA Software Technology Branch was quoted at a 2003 Technical Education Seminar in Chicago as saying "By using VR in education, material now considered too difficult for many students and taught even to advanced learners only at the college level could be mastered by most students in middle school and high school." Virtual reality (VR), the world's most advanced learning technology, is an innovative new way to teach and engage students. It allows students to immerse themselves in 3-D, interactive worlds. The immersive learning system developed here, named TILE (Tactus Immersive Learning Environment), is such a system which extends school boundaries in an economical manner. ${ }^{1}$ Schools that could not previously afford luxuries like science and language labs or field trips now will see students experimenting and exploring in VR at a fraction of the cost. Because the TILE system is built upon the same projectors and PC's already in schools, it is within the means of every school to greatly increase student achievement.

The theoretical foundation for the proposed learning innovation is Cognitive Constructivism. Cognitive Constructivism is a nearly universally accepted learning theory which holds that learning activities that are active, effective and meaningful, result in superior learning versus those that are passive and non-engaging ${ }^{2}$. The primary assertion of the theory is that a person must be fully engaged during the learning process, beyond simply reading, listening to others and/or observing others, for effective learning to occur. Further, three instructional methods that will be facilitated using TILE are cooperative learning, discussion and discovery. Within TILE, students will be able to work together in an immersive environment and discuss what they are learning about. Research supports the effectiveness of cooperative learning and discussion as methods for facilitating academic achievement. Discovery or inquiry-based learning, where information learned through exploration, is also supported by research., ${ }^{3,4,5}$ 
Virtual Reality has received great acceptance in many domains, as it is a truly effective educational and scientific exploration medium. In today's virtual worlds, surgeons cut into computer-simulated anatomy to practice tricky operations. Golfers keep their game up to par in the off-season by teeing up on fake fairways. People plagued by phobias confront their fears by crossing artificial bridges or handling simulated snakes and spiders. Architects walk around in virtual buildings, checking them for accessibility for the handicapped and code violations before a single brick is laid. Military and industry leaders such as GM, Mercedes-Benz, and Shell Oil have extensively relied on VR applications for training and product development. ${ }^{6,7}$ People often associate virtual reality and computer simulations with science fiction, high-tech industries, and computer games, where few associate these technologies with education. Virtual reality and computer simulations have been used in applied fields such as aviation and medicine for years and have recently begun to make their way into the classroom. There is now a sizeable research base addressing the effectiveness of virtual reality and computer simulations within school curriculum. Research demonstrates, for example, that learning geometry through virtual reality has specific advantages over traditional instructional experiences. ${ }^{8,9,10}$ Additional studies found that math and science students who learned through virtual reality were better able to understand abstract concepts and naturally occurring phenomena than students who did not. $^{11,12,13,14,15,16}$ Educational virtual environments have also facilitated leaning in English language arts, foreign language and culture, and social studies. ${ }^{17,18}$ Further studies found that students across various age and grade levels who learned through virtual reality had improved skill sets such as reading problem solving science process skills, 3D visualization, abstract thinking, and creativity. ${ }^{12,19,20,21,22,23,24,25}$ There is also evidence that virtual reality raises interest and motivation in students and effectively supports knowledge transfer. ${ }^{8,9,26,27}$ Based on this prior evidence the TILE system promises to improve educational outcomes for students by delivering a significantly superior mode of instruction. However, there are two major obstacles that must be overcome for immersive VR to be adopted in this arena. The first is cost. The military and big industry have both adopted immersive VR because the investments have a strong business case (a typical immersive VR system might cost well over half a million dollars). In order for immersive VR to be a feasible mode of content delivery in academic institutions, costs would have to be dramatically reduced. The second obstacle is expertise. For immersive VR to be justifiable, a variety of learning scenarios would have to be available, particularly in science. Currently, it takes a highly specialized team of programmers and engineers to develop high quality immersive VR environments.

This project aims to build the foundation for overcoming these two obstacles by demonstrating feasibility for a low cost, easy to use immersive virtual reality platform that includes a hardware and software component. TILE is a system which utilizes inexpensive commodity hardware to overcome the cost obstacle, as well as easy to use authoring and programming environments, so that both non-technical users, such as teachers as well as more technical users, such as computer programmers, can make environments for the TILE platform.

\section{DESCRIPTION OF WORK}

The underlying work conducted here has resulted in a hardware platform and software architecture for immersive environment development across all levels of curricula. The Software Development Toolkit (SDK) and authoring tool enable educational software companies, amateur software developers, teachers and students to create applications supporting their specific needs within this learning environment.

The SDK is basically a set of pre-written software algorithms that can be used by other programs. Effectively what this does is give less experienced or less specialized programmer's access to code written by more specialized or more experienced programmers. The SDK will empower programmers with little or no VR experience to develop immersive applications quickly and easily. The authoring tool will allow non-programmers to design immersive simulations using a standard Windows application interface. This will allow educators and other non-computer programmers to take advantage of this rich learning environment without requiring substantial training and programming experience. The SDK also enables educational software companies to focus their efforts on educational content rather than VR concepts.

In conjunction with participating teachers, we developed two parallel lessons that taught the exact same content, one incorporating VR and the other non-VR. The only difference between the lessons were those qualities that are inherent to VR (e.g., very high levels of interaction) and were not available to the non-VR treatment group. This assured that the differences detected reflect differences in delivery method, not content. The students in both treatments were also assessed using the exact same instruments. 


\section{RESULTS}

We worked with teachers to develop content for use in pre-established classes, therefore we used a Hierarchical Linear Model (HLM) for assigning classes to treatments, which determined school and class effects and therefore provided a more accurate treatment effect. Within each school there were two participating teachers. Each teacher provided both the VR and non-VR lessons using the Motions and Forces virtual environment. This virtual environment enables students to study basic Newtonian physics and experiment with moving objects. For example, students will learn about elastic and inelastic collisions by interacting with two trains in an environment where they control the mass and velocity of each train. They will study the principle of acceleration using a virtual inclined plane where they have the ability to change the mass of each object along with the incline angle and watch the effects of their changes both in action as well as graphically in real time. Newton's law of gravitation is another interactive experiment that allows the user to change the gravitational constant, mass, velocity and altitude of a satellite in space to see how various factors affect the objects orbit. Both teachers taught their regularly assigned classes. Each class's assignment to treatment type was randomized.

Two schools participated in the study. The Motions and Forces software was implemented in each of the schools, along with a parallel non-VR lesson developed for the study. The VR lessons were given to the experimental groups and the non-VR lessons were given to the control groups. This resulted in 180 students enrolled in the study.

Our power analysis was based on data from current research by Virvou, Katsionis and Manos comparing VR to traditional instruction with fourth-grade students learning about geography. ${ }^{28}$ A power analysis based on their effect size of .97 , which would be considered a large effect, indicated sufficient statistical power using two treatments of 45 students each, a total of 90 students. Our study occurred in two schools, with students clustered in four classes in each school, for a total of 180 students. Based on the assumption that a typical class size would be about 20-25 students, our subject pool was in excess of the number of students needed according to the Power Analysis.

To control for contamination among teachers, we requested, subsequent to training and prior to treatments, that teachers in the same buildings/districts not discuss their perceptions of either treatment or either type, emphasizing the compromise to data integrity if this occurred. To minimize student contamination, both treatments in each building were planned for the same day. This limited the non-academic interactions of students (e.g., lunch periods, study halls) and subsequently curtailed any instances of contamination. Moreover, all data from each student was collected during one treatment session thus, further minimizing the possibility of contamination.

An initial pilot validation study was conducted on site at Tactus with students enrolled in typical education classes. This study, referred to in Table 1 as control, demonstrated comparative effectiveness for the TILE system in terms of learning based on an 11 item multiple-choice test, and satisfaction with lesson type based on a 7 item Likert scale, both completed immediately after treatment. Students in both groups were then exposed to the opposite treatment and then completed a 6 item preference survey for lesson format, with each item requiring students to choose between 3D and PowerPoint. This resulted in combined preferences of 52 for TILE and 8 for PowerPoint for the control study.

The next component of the validation study was conducted in classrooms at two local schools. In Table 1 this study is referred to as in-class. Overall this study yielded similar results to the control study conducted at Tactus. However, there was less of a difference found for learning than in the control study. This difference in learning between the TILE and Powerpoint groups was not statistically significant. These inconsistent findings for learning could be attributed to the implementation of the TILE system, rather than the technology itself. For example, the control study was created and performed by researches that were well versed in using the TILE system whereas the in-class study was conducted by teachers who were newly introduced to using the system.

Data that were most consistent across the two studies were those for preference. Similar to the control study, the TILE group chose 3D for 60 items compared to 0 items for PowerPoint, and the PowerPoint Group chose TILE for 52 items compared to 8 items for PowerPoint. This resulted in combined preferences of 112 for TILE and 
8 for PowerPoint for the in-class study. Further, for the two studies combined, preference was indicated for TILE on 164 items compared to 16 for PowerPoint.

Table 1: Validation Study Data

\begin{tabular}{|r|c|c|c|c|c|}
\hline \multicolumn{1}{|c|}{ Group } & Learning & $\begin{array}{c}\text { Satisfaction } \\
\text { Survey }\end{array}$ & & $\begin{array}{c}\text { Preference Survey } \\
\text { TILE (3D)/PowerPoint }\end{array}$ \\
\hline Control & & & & & $29 / 7$ \\
\hline PowerPoint & 7.17 & 2.98 & & & $23 / 1$ \\
\hline TILE (3D) & 8.50 & 4.54 & & & $52 / 8(\mathrm{p}<.001)$ \\
\hline In-Class & 1.33 & 1.56 & & Sub-total & $52 / 8$ \\
\hline Difference & & & & $60 / 0$ \\
\hline PowerPoint & 8.00 & 3.62 & & & $112 / 8(\mathrm{p}<.0001)$ \\
\hline
\end{tabular}

The main result of this research was a prototype one wall interactive 3D immersive learning environment called TILE (Figure 1). This prototype provides educators with a low-cost educational tool that provides rich, interactive, and engaging learning environments that in reality would be too dangerous, too expensive or simply impossible to access. It promises to deliver a viable alternative to conventional teaching methods, to raise interest and motivation of students and in effect increase knowledge transfer and retention. This software can be used as a display for the entire class through the use of a 3D projector or it can be used on an individual basis by students on a standard PC.
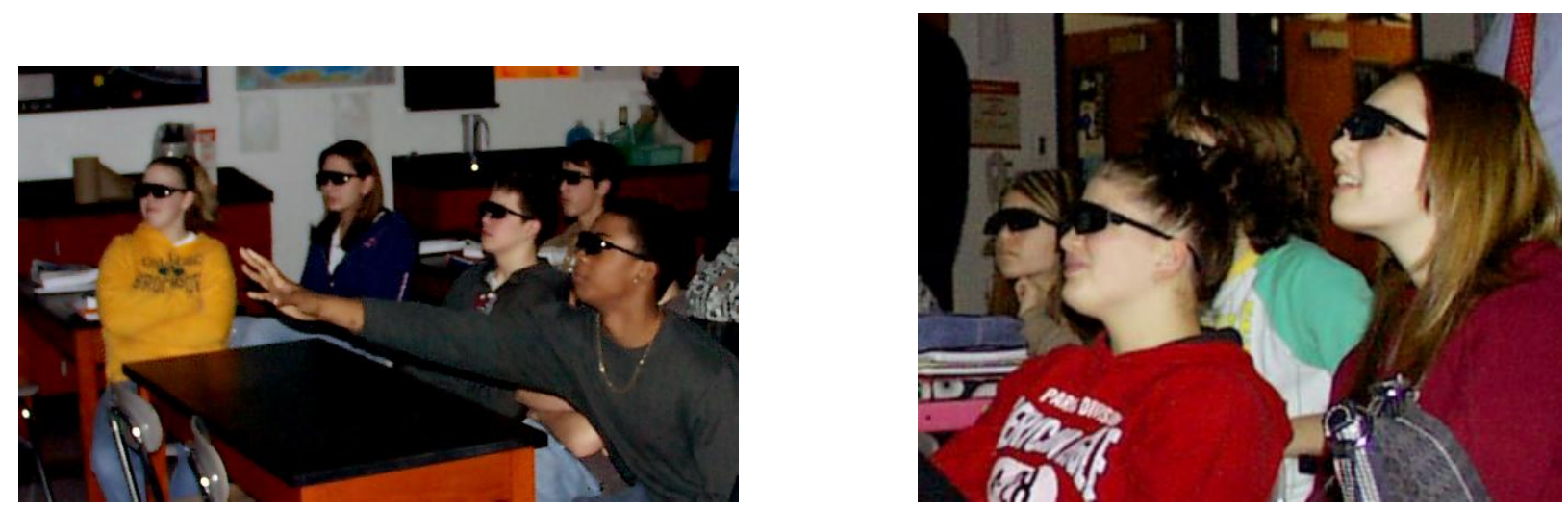

Figure 1: TILE system in-class validation study

An exhaustive testing of commodity equipment was conducted to find the lowest cost, highest fidelity components available which met the technical requirements for TILE. On site testing was conducted on nearly a dozen configurations of hardware and the final design was built and tested. The total hardware cost for the TILE system was around $\$ 5,000$, which includes the cost of a fully functioning graphics computer. This hardware footprint establishes feasibility in terms of both cost and high fidelity functionality. A prototype authoring tool was also developed which allows a novice to design and build an immersive virtual reality application for use with the TILE platform. The user can drag and drop 3D models into the authoring tool and then define their behavior. Additionally, PowerPoint slides and web pages can seamlessly be incorporated with the same drag and drop procedure. This allows a teacher to utilize all of his or her previously developed educational content in these formats in a TILE presentation. This effectively means that a teacher can bring PowerPoint, web, and 3D VR into a lesson with a single piece of software. Secondly, a prototype software developer's kit or SDK was completed. This allows for programmers without specialized VR skills to quickly develop high fidelity immersive VR applications. 3D model loading, content presentation, PowerPoint and web support, text, and audio are all built in, requiring very 
little VR training to develop programs for the TILE platform. The system's usability was also shown to be feasible, both on the content development side, as well as the in classroom deployment side. The TILE system was easy to set up (an average installation took about 20 minutes), it was physically installed in a number of schools, and the platform was used for instruction. On the software side, the Motions and Forces immersive environment was radically easier to develop using the authoring tool than building it from scratch, and was as visually effective as custom built VR applications.

\section{CONCLUSION}

Based on the completed prototype and the preliminary pilot study, we are confident that both efficacy and feasibility have been established. Students seem to both learn better and are more motivated in the immersive environment than with traditional methods. The fact that they were shown a proof of concept immersive environment that was created using the GUI authoring tool demonstrates that a low cost, low expertise immersive VR system is feasible. Future studies need to be performed, on a much larger scale over a longer period of time, where assessment of actual learning and knowledge retention is investigated. These future studies should also be performed, individually, by each student in a computer lab setting so that the interactive nature of the technology is really put to the test. Before a study of this magnitude can be performed, additional 3D interactive software needs to be developed and additional teachers need to be trained in the use of this new technology. The only training necessary for educators in the use of this technology lies on the software side because the hardware is now standard in most schools around the world. 3D content developers are needed to really push this new technology into the classroom and from a close look at the market; companies are slow to develop this type of software. A major push from educators with a desire to implement this type of educational technology in the classroom is needed in order to attract additional companies to this type of software development.

\section{AUTHOR INFORMATION}

Dr. Mayrose received a $\mathrm{PhD}$ in Mechanical Engineering from the University at Buffalo in 2000. He is an Associate Professor of Mechanical Engineering Technology at Buffalo State College and has been conducting research in the fields of Traumatic Injury, Biomechanics, Virtual Reality and Engineering Education for over 15 years. He has published 32 peer reviewed articles and has given 23 presentations at various national/international meetings. Dr. Mayrose has received over $\$ 7,000,000$ in grant funding and was recently awarded "Inventor of the Year" by the Niagara Frontier Intellectual Property Law Association and Technical Societies Council for a US Patent he received. E-mail: mayrosj@buffalostate.edu.

\section{REFERENCES}

1. Tactus Technologies, www.tactustech.com.

2. $\quad$ Gentile, J.R. and J.P. Lalley, Educational Psychology. 2005, Dubuque, IA: Kendall-Hunt.

3. Johnson DW, J.R., Cooperation and competition: Theory and Research. 1989, Edina, MN: Interaction Book Co.

4. Council, N.R., National Science Education Standards: Observe, Interact, Change, Learn. 1995, Washington, DC: National Academis Press.

5. Rosen, J., et al., The Blue Dragon - a System for Monitoring the Kinematics and the Dynamics of Endoscopic Tools in Minimally Invasive Surgery for Objective Laparoscopic Skill Assessment. Studies in Health Technology and Informatics - Medicine Meets Virtual Reality, 2002: p. 412-418.

6. Brown, J.D., et al., In-Vivo and In-Situ Compressive Properties of Porcine Abdominal Soft Tissues. Technology and Informatics: MMVR 2003, 2003. 94: p. 26-32.

7. Raghupathi, L., et al., Simulation of Bleeding During Laparoscopic Herniorrhaphy. Technology and Informatics: MMVR 2002, 2002. 85: p. 382-385.

8. Ottensmeyer, M.P., In Vivo Measurement of Solid Organ Visco-Elastic Properties. Technology and Informatics: MMVR 2002, 2002: p. 328-333.

9. Kesavadas, T., et al. A Virtual Environment for Esophageal Intubation Training. in Medicine Meets Virtual Reality. 2002. Chicago. 
10. Bruyns, C. and K. Montgomery, Generalized Interactions Using Virtual Tools Within the Spring Framework. Technology and Informatics: MMVR 2002, 2002. 85: p. 79-85.

11. Bruyns, C. and K. Montgomery, Generalized Interactions Using Virtual Tools Within the Spring Framework: Probing, Piercing, Cauterizing and Ablating. Technology and Informatics: MMVR 2002, 2002. 85: p. 74-78.

12. Webster, R.W., et al., A Prototype Haptic Suturing Simulator. Technology and Informatics: MMVR 2001, 2001. 81: p. 567-569.

13. Oppenheimer, P., A. Gupta, and S. Weghorst, The Representation of Blood Flow in Endourologic Surgical Simulations. Technology and Informatics: MMVR 2001, 2001: p. 365-371.

14. Yamaguchi, T., Computational Visualization of Cardiovascular Blood Flow. Proceedings of MMVR 1996, 1996: p. 703-712.

15. Mosengard, J., LR-Spring Mass Model for Cardiac Surgical Simulation. Medicine Meets Virtual Reality, 2004.

16. Isabelle, S., Defense Applications of the Cave. 2005.

17. Virtual Reality for Oil and Gas Industry. ERCIM News, 1999(37).

18. Ainge, D.J., Upper Primary Students Constructing and Exploring Three Dimensional Shapes: A Comparison of Virtual Reality with Card Nets. Journal of Educational Computing Research, 1996. 14(4): p. 345-369.

19. Song, K., B. Han, and Y.W. Lee, A Virtual Reality Application for Middle School Geometry Class. 2000.

20. Verzoni, K.A., Creating Simulations: Expressing Life-Situated Relationships in Terms of Algebraic Equations. Annual meeting of the Northeastern Educational Research Association, 1995.

21. Zietsman, A.I. and P.W. Hewson, Effect of Instruction Using Microcomputer Simulations and Conceptual Change Strategies on Science Learning. Journal of Research in Science Teaching, 1986. 1(23): p. 27-39.

22. Jiang, Z. and W.D. Potter, A Computer Microworld to Introduce Students to Probability. Journal of Computers in Mathematics and Science Training, 1994. 13(2): p. 197-222.

23. Kangassalo, M., Children's Independent Exploration of a Natural Phenomenon by Using a Pictoral Computer-Based Simulation. 1994. 3(5): p. 285-297.

24. Stratford, S.J., A Review of Computer-Based Model Research in Precollege Science Classroom. Journal of Computers in Mathematics and Science Teaching, 1997. 16(1): p. 3-23.

25. Gorsky, P. and M. Finegold, Using Computer Simulations to Restructure Students' Conceptions of Force. Journal of Computers in Mathematics and Science Teaching, 1992. 1(11): p. 163-178.

26. Yair, Y., R. Mintz, and S. Litvak, 3-D Virtual Reality in Science Education: An Implication for Astronomy Teaching. Journal of Computers in Mathematics and Science Education, 2001. 20(3): p. 293-301.

27. Schwienhorst, K., Why Virtual, Why Environments? Implementing Virtual Reality Concepts in ComputerAssisted Language Learning. Simulation \& Gaming, 2002. 33(2): p. 196-209.

28. Virvou, M., Katsionis, G., \& Manos, K., Combining Software Games with Education: Evaluation of its Educational Effectiveness. Educational Technology \& Society, 2005, 8 (2), 54-65. 